\title{
Sharp Shafer-Fink type inequalities for Gauss lemniscate functions
}

\author{
Ji-En Deng ${ }^{*}$ and Chao-Ping Chen
}

\author{
"Correspondence: \\ dengjien454000@sohu.com \\ School of Mathematics and \\ Informatics, Henan Polytechnic \\ University, Jiaozuo, Henan 454000, \\ China
}

\begin{abstract}
In this paper, we establish sharp Shafer-Fink type inequalities for Gauss lemniscate functions.
\end{abstract}

MSC: 26007

Keywords: inequalities; lemniscate functions

\section{Introduction and definitions}

In geometry, the lemniscate of Bernoulli is a plane curve defined by two given points $F_{1}$ and $F_{2}$, known as foci, at distance $2 a$ from each other as the locus of points $P$ so that $P F_{1} \cdot P F_{2}=a^{2}$. This gives the equation $\left(x^{2}+y^{2}\right)^{2}=2 a^{2}\left(x^{2}-y^{2}\right)$. In polar coordinates $(r, \theta)$, the equation becomes $r^{2}=2 a \cos (2 \theta)$. The arc length from the origin to a point on the Bernoulli lemniscate $r^{2}=\cos (2 \theta)$ is given by the function

$$
\operatorname{arcsl} x=\int_{0}^{x} \frac{\mathrm{d} t}{\sqrt{1-t^{4}}}, \quad|x| \leq 1,
$$

where $\operatorname{arcsl} x$ is called the arc lemniscate sine function studied by CF Gauss in 1797-1798. Another lemniscate function investigated by Gauss is the hyperbolic arc lemniscate sine function, defined as

$$
\operatorname{arcslh} x=\int_{0}^{x} \frac{\mathrm{d} t}{\sqrt{1+t^{4}}}, \quad x \in \mathbb{R} .
$$

The functions (1.1) and (1.2) can be found in [1, p.259], [2, (2.5)-(2.6)], [3-7] and [8, Ch. 1].

Following Neuman [3], Gauss' arc lemniscate tangent and the hyperbolic arc lemniscate tangent functions are defined by

$$
\operatorname{arctl} x=\operatorname{arcsl}\left(\frac{x}{\sqrt[4]{1+x^{4}}}\right), \quad x \in \mathbb{R}
$$

and

$$
\operatorname{arctlh} x=\operatorname{arcslh}\left(\frac{x}{\sqrt[4]{1-x^{4}}}\right), \quad|x|<1,
$$

respectively.

(2014 Deng and Chen; licensee Springer. This is an Open Access article distributed under the terms of the Creative Commons Attribution License (http://creativecommons.org/licenses/by/2.0), which permits unrestricted use, distribution, and reproduction in any medium, provided the original work is properly cited. 
For $0 \leq x \leq 1$, it is known in the literature that

$$
\frac{3 x}{2+\sqrt{1-x^{2}}} \leq \frac{6(\sqrt{1+x}-\sqrt{1-x})}{4+\sqrt{1+x}+\sqrt{1-x}} \leq \arcsin x \leq \frac{\pi x}{2+\sqrt{1-x^{2}}} .
$$

The first and second inequalities in equation (1.5) were established by Shafer (see, e.g., [9, p.247]), while the third inequality was proved by Fink [10]. In recent years, ShaferFink's inequalities have attracted much attention of the mathematical community. By using the $\lambda$-method of Mitrinović and Vasić [9], Malešević [11] improved the upper bound for $\arcsin x$ and established the following inequality: For $0 \leq x \leq 1$,

$$
\frac{3 x}{2+\sqrt{1-x^{2}}} \leq \arcsin x \leq \frac{(\pi /(\pi-2)) x}{(2 /(\pi-2))+\sqrt{1-x^{2}}} \leq \frac{\pi x}{2+\sqrt{1-x^{2}}} .
$$

In [12-15], other upper bounds for $\arcsin x$ were established: For $0 \leq x \leq 1$,

$$
\begin{aligned}
\frac{3 x}{2+\sqrt{1-x^{2}}} & \leq \frac{6(\sqrt{1+x}-\sqrt{1-x})}{4+\sqrt{1+x}+\sqrt{1-x}} \leq \arcsin x \\
& \leq \frac{(\pi(2-\sqrt{2}) /(\pi-2 \sqrt{2}))(\sqrt{1+x}-\sqrt{1-x})}{(\sqrt{2}(4-\pi) /(\pi-2 \sqrt{2}))+\sqrt{1+x}+\sqrt{1-x}} \\
& \leq \frac{\pi(\sqrt{2}+1 / 2)(\sqrt{1+x}-\sqrt{1-x})}{4+\sqrt{1+x}+\sqrt{1-x}} \leq \frac{\pi x}{2+\sqrt{1-x^{2}}} .
\end{aligned}
$$

In [16], Pan and Zhu gave some further generalizations of these results and obtained two new Shafer-Fink type double inequalities. In [17], Zhu provided a solution to an open problem posed by Oppenheim in [18]. At the same time, some Shafer-Fink inequalities were deduced from the solution of Oppenheim's problem. Chen and Cheung [19] provided a laconic proof to Oppenheim's problem. Recently, Qi and Guo [20,21] presented a sharpening and generalizations of Shafer-Fink's inequality.

Related to the inverse sine inequality, the inverse tangent inequality is also of much interest. In the literature, we have

$$
\frac{3 x}{1+2 \sqrt{1+x^{2}}}<\arctan x<\frac{2 x}{1+\sqrt{1+x^{2}}} \text { for } x>0 .
$$

The first inequality in equation (1.8) was presented without proof by Shafer [22]. Three proofs of it were later given in [23]. The second inequality in equation (1.8) can be found in, e.g., [24, p.288]. Shafer's inequality (1.8) was recently sharpened and generalized by Qi et al. in [25]. For each $\theta>0$, Chen et al. [26] determined the largest number $\theta_{1}$ and the smallest number $\theta_{2}$ such that the inequalities

$$
\frac{\theta_{1} x}{1+\theta \sqrt{1+x^{2}}} \leq \arctan x \leq \frac{\theta_{2} x}{1+\theta \sqrt{1+x^{2}}}
$$

are valid for all $x \geq 0$. Zhu [27, Theorems 1.9 and 1.10] established Shafer-Fink type inequalities for the inverse hyperbolic sine function.

Recently, numerous inequalities have been given for the lemniscate functions. For example, Neuman [5] proved the following inequalities:

$$
\left(\frac{5}{3+2\left(1-x^{4}\right)^{1 / 2}}\right)^{1 / 2}<\frac{\operatorname{arcsl} x}{x}<\left(1-x^{4}\right)^{-1 / 10}
$$


and

$$
\left(\frac{5}{3+2\left(1+x^{4}\right)^{1 / 2}}\right)^{1 / 2}<\frac{\operatorname{arcslh} x}{x}<\left(1+x^{4}\right)^{-1 / 10}
$$

for $0<|x|<1$.

Chen [28, 29] established Wilker and Huygens type inequalities for Gauss lemniscate functions. For example, Chen [28] proved that for $0<|x|<1$,

$$
2+\frac{1}{20} x^{3} \operatorname{arctl} x<\left(\frac{\operatorname{arcsl} x}{x}\right)^{2}+\frac{\operatorname{arctl} x}{x}
$$

with the best possible constant $\frac{1}{20}$. Chen [29] proved that for $0<|x|<1$,

$$
\frac{2(\operatorname{arcsl} x / x)+\operatorname{arctl} x / x}{3}>\frac{3(\operatorname{arcsl} x / x)+2(\operatorname{arctl} x / x)}{5}>1
$$

In this paper, we establish sharp Shafer-Fink type inequalities for Gauss lemniscate functions.

The following lemma is required in our present investigation.

Lemma 1.1 ([30-32]) Let $-\infty<a<b<\infty$, and let $f, g:[a, b] \rightarrow \mathbb{R}$ be continuous on $[a, b]$, differentiable on $(a, b)$. Let $g^{\prime}(x) \neq 0$ on $(a, b)$. Iff $(x) / g^{\prime}(x)$ is increasing (decreasing) on $(a, b)$, then so are

$$
[f(x)-f(a)] /[g(x)-g(a)] \text { and }[f(x)-f(b)] /[g(x)-g(b)] .
$$

If $f^{\prime}(x) / g^{\prime}(x)$ is strictly monotone, then the monotonicity in the conclusion is also strict.

Remark 1.1 A generalization of the familiar trigonometric and hyperbolic functions was described by Lindqvist [33]. The generalized $p$-trigonometric functions occur as an eigenfunction of the Dirichlet problem for the one-dimensional $p$-Laplacian. Recently, the $p$ trigonometric functions have been studied extensively, see for example [34-37] and their references. Very recently, Takeuchi [38] (see also [39]) introduced the ( $p, q)$-trigonometric functions that coincide with the $p$-trigonometric functions for $p=q$ and are connected with the Dirichlet problem for the $p, q$-Laplacian. These $(p, q)$-trigonometric functions have been the subject of intense investigations (see, for example, [35, 39-42]). For $p, q>1$ the function $\arcsin _{p, q}$ is defined in $[38,39]$ by

$$
\arcsin _{p, q}(x)=\int_{0}^{x} \frac{\mathrm{d} t}{\left(1-t^{q}\right)^{1 / p}}, \quad|x| \leq 1 .
$$

Similarly, for $p, q>1$ the function $\operatorname{arcsinh}_{p, q}$ is defined by [40]

$$
\operatorname{arcsinh}_{p, q}(x)=\int_{0}^{x} \frac{\mathrm{d} t}{\left(1+t^{q}\right)^{1 / p}}, \quad x \geq 0 .
$$

Clearly,

$$
\operatorname{arcsl} x=\arcsin _{2,4}(x) \text { and } \operatorname{arcslh} x=\operatorname{arcsinh}_{2,4}(x) \text {. }
$$




\section{Main results}

Theorem 2.1 For $0<x<1$,

$$
\frac{\alpha_{1}(\sqrt{1+x}-\sqrt{1-x})}{4-\sqrt{1+x}-\sqrt{1-x}}<\operatorname{arcsl} x<\frac{\beta_{1}(\sqrt{1+x}-\sqrt{1-x})}{4-\sqrt{1+x}-\sqrt{1-x}}
$$

with the best possible constants

$$
\alpha_{1}=2 \quad \text { and } \quad \beta_{1}=\frac{2 \sqrt{2}-1}{4} B\left(\frac{1}{4}, \frac{1}{2}\right)=2.39712057 \ldots
$$

Here

$$
B(x, y)=\int_{0}^{1} t^{x-1}(1-t)^{y-1} \mathrm{~d} t
$$

is the beta function.

Proof For $0<x<1$, let

$$
f(x)=\frac{f_{1}(x)}{f_{2}(x)}
$$

where

$$
f_{1}(x)=\operatorname{arcsl} x \text { and } f_{2}(x)=\frac{\sqrt{1+x}-\sqrt{1-x}}{4-\sqrt{1+x}-\sqrt{1-x}}
$$

Then,

$$
\frac{2 f_{1}^{\prime}(x)}{f_{2}^{\prime}(x)}=\frac{(4-\sqrt{1+x}-\sqrt{1-x})^{2} \sqrt{1+x} \sqrt{1-x}}{\sqrt{1-x^{4}}(-1+\sqrt{1-x}+\sqrt{1+x})}=: f_{3}(x) .
$$

Differentiation yields

$$
f_{3}^{\prime}(x)=\frac{4-\sqrt{1+x}-\sqrt{1-x}}{\sqrt{1-x^{2}} \sqrt{1-x^{4}}\left(x^{2}+1\right)(-1+\sqrt{1-x}+\sqrt{1+x})^{2}} f_{4}(x),
$$

where

$$
\begin{aligned}
f_{4}(x)= & \left(6 x^{3}+x^{2}-4 x+1\right) \sqrt{1-x}+\left(6 x^{3}-x^{2}-4 x-1\right) \sqrt{1+x} \\
& -\left(x^{3}-3 x\right) \sqrt{1-x^{2}}-6 x^{3}+6 x
\end{aligned}
$$

Motivated by the investigations in [12], we are in a position to prove $f_{4}(x)>0$ for $x \in(0,1)$.

Let

$$
A(x)= \begin{cases}\mu, & x=0, \\ \frac{f_{4}(x)}{x^{3}}, & 0<x<1, \\ 0, & x=1,\end{cases}
$$


where $\mu$ is constant determined with limit:

$$
\mu=\lim _{x \rightarrow 0} \frac{f_{4}(x)}{x^{3}}=\frac{27}{8} .
$$

Using Maple we determine Taylor approximation for the function $A(x)$ by the polynomial of the fourth order:

$$
P_{1}(x)=\frac{27}{8}-\frac{159}{128} x^{2}-\frac{465}{1,024} x^{4}
$$

which has a bound of absolute error

$$
\varepsilon_{1}=\frac{1,719}{1,024}
$$

for values $x \in[0,1]$. It is true that

$$
A(x)-\left(P_{1}(x)-\varepsilon_{1}\right) \geq 0, \quad 0 \leq x \leq 1
$$

and

$$
P_{1}(x)-\varepsilon_{1}=\frac{1,737}{1,024}-\frac{159}{128} x^{2}-\frac{465}{1,024} x^{4}>0, \quad 0<x<1 .
$$

Hence, for $x \in(0,1)$ it is true that $A(x)>0$ and therefore $f_{4}(x)>0$ and $f_{3}^{\prime}(x)>0$ for $x \in(0,1)$. Therefore, the function $\frac{f_{1}^{\prime}(x)}{f_{2}^{\prime}(x)}$ is strictly increasing on $(0,1)$. By Lemma 1.1, the function

$$
f(x)=\frac{f_{1}(x)}{f_{2}(x)}=\frac{f_{1}(x)-f_{1}(0)}{f_{2}(x)-f_{2}(0)}
$$

is strictly increasing on $(0,1)$. And hence,

$$
2=\lim _{x \rightarrow 0} f(x)<f(x)=\frac{\operatorname{arcsl} x}{\frac{\sqrt{1+x}-\sqrt{1-x}}{4-\sqrt{1+x}-\sqrt{1-x}}}<\lim _{x \rightarrow 1} f(x)=\frac{2 \sqrt{2}-1}{4} B\left(\frac{1}{4}, \frac{1}{2}\right)
$$

for $0<x<1$. By rearranging terms in the last expression, Theorem 2.1 follows.

Theorem 2.2 For $0<x<1$,

$$
\frac{\alpha_{2}(\sqrt{1+x}-\sqrt{1-x})}{4-\sqrt{1+x}-\sqrt{1-x}}<\operatorname{arctlh} x<\frac{\beta_{2}(\sqrt{1+x}-\sqrt{1-x})}{4-\sqrt{1+x}-\sqrt{1-x}}
$$

with the best possible constants

$$
\alpha_{2}=2 \quad \text { and } \quad \beta_{2}=\frac{2 \sqrt{2}-1}{4} B\left(\frac{1}{4}, \frac{1}{4}\right)=3.39004 \ldots
$$

Here $B(x, y)$ denotes the beta function.

Proof For $0<x<1$, let

$$
F(x)=\frac{F_{1}(x)}{F_{2}(x)}
$$


where

$$
F_{1}(x)=\operatorname{arctlh} x \quad \text { and } \quad F_{2}(x)=\frac{\sqrt{1+x}-\sqrt{1-x}}{4-\sqrt{1+x}-\sqrt{1-x}} .
$$

Then,

$$
\frac{2 F_{1}^{\prime}(x)}{F_{2}^{\prime}(x)}=\frac{(4-\sqrt{1+x}-\sqrt{1-x})^{2} \sqrt{1+x} \sqrt{1-x}}{\left(1-x^{4}\right)^{3 / 4}(-1+\sqrt{1+x}+\sqrt{1-x})}=: F_{3}(x) .
$$

Differentiation yields

$$
F_{3}^{\prime}(x)=\frac{4-\sqrt{1+x}-\sqrt{1-x}}{\sqrt{1-x} \sqrt{1+x}(-1+\sqrt{1-x}+\sqrt{1+x})^{2}\left(1-x^{4}\right)^{3 / 4}\left(x^{2}+1\right)} F_{4}(x),
$$

where

$$
\begin{aligned}
F_{4}(x)= & \left(11 x^{3}-x^{2}-4 x-1\right) \sqrt{1+x}+\left(11 x^{3}+x^{2}-4 x+1\right) \sqrt{1-x} \\
& -3 x\left(x^{2}-1\right) \sqrt{1-x^{2}}-12 x^{3}+6 x .
\end{aligned}
$$

Motivated by the investigations in [12], we are in a position to prove $F_{4}(x)>0$ for $x \in(0,1)$.

Let

$$
B(x)= \begin{cases}\lambda, & x=0, \\ \frac{F_{4}(x)}{x^{3}}, & 0<x<1, \\ 5 \sqrt{2}-6, & x=1,\end{cases}
$$

where $\lambda$ is a constant determined by the limit

$$
\lambda=\lim _{x \rightarrow 0} \frac{F_{4}(x)}{x^{3}}=\frac{43}{8} .
$$

Using Maple we determine a Taylor approximation for the function $B(x)$ by the polynomial of fourth order:

$$
P_{2}(x)=\frac{43}{8}-\frac{191}{128} x^{2}-\frac{609}{1,024} x^{4},
$$

which has a bound of the absolute error of

$$
\varepsilon_{2}=\frac{9,511}{1,024}-5 \sqrt{2}
$$

for values $x \in[0,1]$. It is true that

$$
B(x)-\left(P_{2}(x)-\varepsilon_{2}\right) \geq 0, \quad 0 \leq x \leq 1
$$

and

$$
P_{2}(x)-\varepsilon_{2}=-\frac{4,007}{1,024}+5 \sqrt{2}-\frac{191}{128} x^{2}-\frac{609}{1,024} x^{4}>0, \quad 0 \leq x \leq 1 .
$$


Hence, for $x \in(0,1)$ it is true that $B(x)>0$ and therefore $F_{4}(x)>0$ and $F_{3}^{\prime}(x)>0$ for $x \in(0,1)$. Therefore, the function $\frac{F_{1}^{\prime}(x)}{F_{2}^{\prime}(x)}$ is strictly increasing on $(0,1)$. By Lemma 1.1, the function

$$
F(x)=\frac{F_{1}(x)}{F_{2}(x)}=\frac{F_{1}(x)-F_{1}(0)}{F_{2}(x)-F_{2}(0)}
$$

is strictly increasing on $(0,1)$. And hence,

$$
2=\lim _{x \rightarrow 0} F(x)<F(x)=\frac{\operatorname{arctlh} x}{\frac{\sqrt{1+x}-\sqrt{1-x}}{4-\sqrt{1+x}-\sqrt{1-x}}}<\lim _{x \rightarrow 1} F(x)=\frac{2 \sqrt{2}-1}{4} B\left(\frac{1}{4}, \frac{1}{4}\right)
$$

for $0<x<1$. By rearranging terms in the last expression, Theorem 2.2 follows.

Theorem 2.3 For $0<|x|<1$,

$$
\frac{a_{1}}{4+\sqrt{1-x^{4}}}<\frac{\operatorname{arcs} 1 x}{x}<\frac{b_{1}}{4+\sqrt{1-x^{4}}}
$$

and

$$
\frac{a_{2}}{\frac{7}{3}+\left(1-x^{4}\right)^{1 / 3}}<\frac{\operatorname{arcsl} x}{x}<\frac{b_{2}}{\frac{7}{3}+\left(1-x^{4}\right)^{1 / 3}}
$$

with the best possible constants

$$
a_{1}=5, \quad b_{1}=B\left(\frac{1}{4}, \frac{1}{2}\right)=5.2441151 \ldots
$$

and

$$
a_{2}=\frac{7}{12} B\left(\frac{1}{4}, \frac{1}{2}\right)=3.0590671 \ldots, \quad b_{2}=\frac{10}{3}=3.3333333 \ldots
$$

Here $B(x, y)$ denotes the beta function.

Proof For $0<x<1$, let

$$
L_{1}(x)=\frac{4+\sqrt{1-x^{4}}}{x} \operatorname{arcsl} x
$$

Differentiation yields

$$
L_{1}^{\prime}(x)=\frac{x^{4}+4 \sqrt{1-x^{4}}+1}{x^{2} \sqrt{1-x^{4}}} L_{2}(x),
$$

where

$$
L_{2}(x)=-\operatorname{arcsl} x+\frac{\left(4+\sqrt{1-x^{4}}\right) x}{x^{4}+4 \sqrt{1-x^{4}}+1} .
$$

Elementary calculation shows that

$$
L_{2}^{\prime}(x)=\frac{24 x^{4}\left(1-\sqrt{1-x^{4}}\right)}{\left(x^{4}+4 \sqrt{1-x^{4}}+1\right)^{2} \sqrt{1-x^{4}}}>0, \quad 0<x<1 .
$$


Hence, $L_{2}(x)>L_{2}(0)=0$ and $L_{1}^{\prime}(x)>0$ for $0<x<1$. Therefore, the function $L_{1}(x)$ is strictly increasing on $(0,1)$. And hence,

$$
5=\lim _{x \rightarrow 0} L_{1}(x)<L_{1}(x)=\frac{4+\sqrt{1-x^{4}}}{x} \operatorname{arcsl} x<\lim _{x \rightarrow 1} L_{1}(x)=B\left(\frac{1}{4}, \frac{1}{2}\right)
$$

for $0<x<1$. Hence, inequality (2.5) holds with the best possible constants given in equation $(2.7)$

For $0<x<1$, let

$$
M_{1}(x)=\frac{\frac{7}{3}+\left(1-x^{4}\right)^{1 / 3}}{x} \operatorname{arcsl} x .
$$

Differentiation yields

$$
M_{1}^{\prime}(x)=\frac{x^{4}+7\left(1-x^{4}\right)^{2 / 3}+3}{3 x^{2}\left(1-x^{4}\right)^{2 / 3}} M_{2}(x)
$$

where

$$
M_{2}(x)=-\operatorname{arcsl} x+\frac{x\left(1-x^{4}\right)^{1 / 6}\left(7+3\left(1-x^{4}\right)^{1 / 3}\right)}{x^{4}+7\left(1-x^{4}\right)^{2 / 3}+3} .
$$

Elementary calculation shows that

$$
M_{2}^{\prime}(x)=-\frac{2 x^{4}}{3\left(1-x^{4}\right)^{3 / 2}\left(x^{4}+7\left(1-x^{4}\right)^{2 / 3}+3\right)^{2}} M_{3}(x),
$$

where

$$
M_{3}(x)=3 x^{8}-66 x^{4}+63+\left(84-56 x^{4}\right)\left(1-x^{4}\right)^{2 / 3}-147\left(1-x^{4}\right)^{4 / 3} .
$$

We claim that $M_{3}(x)>0$ for $0<x<1$. By an elementary change of variable

$$
x=\left(1-t^{3}\right)^{1 / 4}, \quad 0<t<1,
$$

we find that

$$
M_{3}(x)>0 \quad \text { for } 0<x<1 \quad \Longleftrightarrow \quad M_{4}(t)>0 \quad \text { for } 0<t<1 \text {, }
$$

where

$$
M_{4}(t)=28 t^{2}+60 t^{3}+56 t^{5}+3 t^{6}-147 t^{4}=t^{2}(1-t)\left(-3 t^{3}-59 t^{2}+88 t+28\right) .
$$

Obviously, $M_{4}(t)>0$ for $0<t<1$. This proves the claim.

Hence, $M_{2}^{\prime}(x)<0$ for $0<x<1$. This implies that $M_{2}(x)<M_{2}(0)=0$ and $M_{1}^{\prime}(x)<0$ for $0<x<1$. Therefore, the function $M_{1}(x)$ is strictly decreasing on $(0,1)$. And hence,

$$
\frac{7}{12} B\left(\frac{1}{4}, \frac{1}{2}\right)=\lim _{x \rightarrow 1} M_{1}(x)<M_{1}(x)=\frac{\frac{7}{3}+\left(1-x^{4}\right)^{1 / 3}}{x} \operatorname{arcsl} x<\lim _{x \rightarrow 0} M_{1}(x)=\frac{10}{3}
$$


for $0<x<1$. Hence, inequality (2.6) holds with the best possible constants given in equation (2.8).

Remark 2.1 (i) There is no strict comparison between the two lower bounds in equations (2.5) and (2.6). Also, there is no strict comparison between the two upper bounds in equations (2.5) and (2.6).

(ii) The lower bound in equation (1.10) is sharper than the one in equation (2.5), since

$$
\frac{5}{3+2\left(1-x^{4}\right)^{1 / 2}}-\left(\frac{5}{4+\sqrt{1-x^{4}}}\right)^{2}=\frac{5\left(1-\sqrt{1-x^{4}}\right)^{2}}{\left(4+\sqrt{1-x^{4}}\right)^{2}\left(3+2 \sqrt{1-x^{4}}\right)}>0, \quad 0<|x|<1 .
$$

There is no strict comparison between the two upper bounds in equations (1.10) and (2.5).

(iii) By two elementary changes of variable,

$$
t=1-x^{4}, \quad 0<x<1 \quad \text { and } t=u^{30}, \quad 0<u<1,
$$

we find that

$$
\begin{aligned}
\frac{\frac{10}{3}}{\frac{7}{3}+\left(1-x^{4}\right)^{1 / 3}}-\frac{1}{\left(1-x^{4}\right)^{1 / 10}} \\
=\frac{10 t^{1 / 10}-7-3 t^{1 / 3}}{\left(7+3 t^{1 / 3}\right) t^{1 / 10}}=\frac{10 u^{3}-7-3 u^{10}}{\left(7+3 u^{10}\right) u^{3}} \\
=-\frac{\left(3 u^{8}+6 u^{7}+9 u^{6}+12 u^{5}+15 u^{4}+18 u^{3}+21 u^{2}+14 u+7\right)(1-u)^{2}}{\left(7+3 u^{10}\right) u^{3}}<0 .
\end{aligned}
$$

Hence, the upper bound in equation (2.6) is sharper than the one in equation (1.10). There is no strict comparison between the two lower bounds in equations (1.10) and (2.6).

Theorem 2.4 For $x \neq 0$,

$$
\frac{a}{\frac{17}{3}+\left(1+x^{4}\right)^{1 / 4}}<\frac{\operatorname{arcslh} x}{x}<\frac{b}{\frac{17}{3}+\left(1+x^{4}\right)^{1 / 4}}
$$

with the best possible constants

$$
a=\frac{1}{4} B\left(\frac{1}{4}, \frac{1}{4}\right)=1.8540746 \ldots \quad \text { and } \quad b=\frac{20}{3}=6.6666666 \ldots
$$

Here $B(x, y)$ denotes the beta function.

Proof The inequality (2.10) is obtained by considering the function $p(x)$ defined by

$$
p(x)=\frac{\left(\frac{17}{3}+\left(1+x^{4}\right)^{1 / 4}\right)}{x} \operatorname{arcslh} x, \quad x>0 .
$$

Differentiation yields

$$
p^{\prime}(x)=-\frac{17\left(1+x^{4}\right)^{3 / 4}+3}{3 x^{2}\left(1+x^{4}\right)^{3 / 4}} \operatorname{arcslh} x+\frac{\left(\frac{17}{3}+\left(1+x^{4}\right)^{1 / 4}\right)}{x} \frac{1}{\sqrt{1+x^{4}}}=\frac{17\left(1+x^{4}\right)^{3 / 4}+3}{3 x^{2}\left(1+x^{4}\right)^{3 / 4}} q(x),
$$


where

$$
q(x)=-\operatorname{arcslh} x+\frac{x\left(1+x^{4}\right)^{1 / 4}\left(17+3\left(1+x^{4}\right)^{1 / 4}\right)}{17\left(1+x^{4}\right)^{3 / 4}+3} .
$$

Elementary calculation shows that

$$
q^{\prime}(x)=-\frac{x^{4}}{\left(1+x^{4}\right)^{3 / 2}\left(17\left(1+x^{4}\right)^{3 / 4}+3\right)^{2}} r(x),
$$

where

$$
r(x)=-27-27 x^{4}-51\left(1+x^{4}\right)^{3 / 4}+578\left(1+x^{4}\right)^{3 / 2} .
$$

We claim that $r(x)>0$ for $x>0$. By an elementary change of variable

$$
t=\left(1+x^{4}\right)^{1 / 4}, \quad x>0,
$$

we find that

$$
r(x)>0 \quad \text { for } x>0 \quad \Longleftrightarrow s(t)>0 \quad \text { for } t>1,
$$

where

$$
s(t)=t^{3}\left(-51-27 t+578 t^{3}\right), \quad t>1 .
$$

Obviously, $s(t)>0$ for $t>1$. This proves the claim.

Hence, $q^{\prime}(x)<0$ and $q(x)<q(0)=0$ for $x>0$. Therefore, $p^{\prime}(x)<0$ for $x>0$, and we have

$$
\frac{1}{4} B\left(\frac{1}{4}, \frac{1}{4}\right)=\lim _{x \rightarrow \infty} p(x)<p(x)=\frac{\left(\frac{17}{3}+\left(1+x^{4}\right)^{1 / 4}\right) \operatorname{arcslh} x}{x}<\lim _{x \rightarrow 0} p(x)=\frac{20}{3} .
$$

Hence, the inequality (2.10) holds with the best possible constants given in equation (2.11).

Remark 2.2 Inequality (1.11) is sharper than inequality (2.10).

Theorem 2.5 For $x \neq 0$,

$$
\frac{\alpha}{\frac{2}{3}+\left(1+x^{4}\right)^{1 / 4}}<\frac{\operatorname{arctl} x}{x}<\frac{\beta}{\frac{2}{3}+\left(1+x^{4}\right)^{1 / 4}}
$$

with the best possible constants

$$
\alpha=\frac{1}{4} B\left(\frac{1}{4}, \frac{1}{2}\right)=1.3110287 \ldots \text { and } \quad \beta=\frac{5}{3}=1.6666666 \ldots
$$

Here $B(x, y)$ denotes the beta function. 
Proof The inequality (2.13) is obtained by considering the function $P(x)$ defined by

$$
P(x)=\frac{\left(\frac{2}{3}+\left(1+x^{4}\right)^{1 / 4}\right)}{x} \operatorname{arctl} x, \quad x>0 .
$$

Differentiation yields

$$
P^{\prime}(x)=-\frac{2\left(1+x^{4}\right)^{3 / 4}+3}{3 x^{2}\left(1+x^{4}\right)^{3 / 4}} \operatorname{arctl} x+\frac{\left(\frac{2}{3}+\left(1+x^{4}\right)^{1 / 4}\right)}{x} \frac{1}{\left(1+x^{4}\right)^{3 / 4}}=\frac{2\left(1+x^{4}\right)^{3 / 4}+3}{3 x^{2}\left(1+x^{4}\right)^{3 / 4}} Q(x),
$$

where

$$
Q(x)=-\operatorname{arctl} x+\frac{\left(2+3\left(1+x^{4}\right)^{1 / 4} x\right.}{2\left(1+x^{4}\right)^{3 / 4}+3} .
$$

Elementary calculation shows that

$$
Q^{\prime}(x)=-\frac{6 x^{4}\left(2 \sqrt{1+x^{4}}+\left(1+x^{4}\right)^{3 / 4}-3\right)}{\left(1+x^{4}\right)^{3 / 4}\left(2\left(1+x^{4}\right)^{3 / 4}+3\right)^{2}}<0, \quad x>0 .
$$

Hence, $Q(x)<Q(0)=0$ for $x>0$. Therefore, $P^{\prime}(x)<0$ for $x>0$, and we have

$$
\frac{1}{4} B\left(\frac{1}{4}, \frac{1}{2}\right)=\lim _{x \rightarrow \infty} P(x)<P(x)=\frac{\left(\frac{2}{3}+\left(1+x^{4}\right)^{1 / 4}\right)}{x} \operatorname{arctl} x<\lim _{x \rightarrow 0} P(x)=\frac{5}{3} .
$$

Hence, the inequality (2.13) holds with the best possible constants given in equation (2.14).

Theorem 2.6 For $0<|x|<1$,

$$
\frac{a_{3}}{\frac{7}{3}+\sqrt{1-x^{4}}}<\frac{\operatorname{arcth} x}{x}<\frac{b_{3}}{\frac{7}{3}+\sqrt{1-x^{4}}}
$$

and

$$
\frac{a_{4}}{\frac{2}{3}+\left(1-x^{4}\right)^{1 / 4}}<\frac{\operatorname{arctlh} x}{x}<\frac{b_{4}}{\frac{2}{3}+\left(1-x^{4}\right)^{1 / 4}}
$$

with the best possible constants

$$
a_{3}=\frac{10}{3}=3.3333333 \ldots, \quad b_{3}=\frac{7}{12} B\left(\frac{1}{4}, \frac{1}{4}\right)=4.3261742 \ldots
$$

and

$$
a_{4}=\frac{1}{6} B\left(\frac{1}{4}, \frac{1}{4}\right)=1.236049 \ldots, \quad b_{4}=\frac{5}{3}=1.666666 \ldots
$$

Proof For $0<x<1$, let

$$
J_{1}(x)=\frac{\frac{7}{3}+\sqrt{1-x^{4}}}{x} \operatorname{arcth} x .
$$


Differentiation yields

$$
J_{1}^{\prime}(x)=\frac{3 x^{4}+7 \sqrt{1-x^{4}}+3}{3 x^{2} \sqrt{1-x^{4}}} J_{2}(x)
$$

where

$$
J_{2}(x)=-\operatorname{arctlh} x+\frac{\left(7+3 \sqrt{1-x^{4}}\right) x}{\left(1-x^{4}\right)^{1 / 4}\left(3 x^{4}+7 \sqrt{1-x^{4}}+3\right)} .
$$

Elementary calculation shows that

$$
J_{2}^{\prime}(x)=\frac{3 x^{4}}{\left(1-x^{4}\right)^{7 / 4}\left(3 x^{4}+7 \sqrt{1-x^{4}}+3\right)^{2}} J_{3}(x),
$$

where

$$
J_{3}(x)=-25 x^{4}-3 x^{8}+28+\left(42 x^{4}-28\right) \sqrt{1-x^{4}} .
$$

We claim that $J_{3}(x)>0$ for $0<x<1$. By an elementary change of variable

$$
x=\left(1-t^{2}\right)^{1 / 4}, \quad 0<t<1,
$$

we find that

$$
J_{3}(x)>0 \quad \text { for } 0<x<1 \quad \Longleftrightarrow \quad J_{4}(t)>0 \quad \text { for } 0<t<1 \text {, }
$$

where

$$
J_{4}(t)=14 t+31 t^{2}-42 t^{3}-3 t^{4}=t(1-t)\left(3 t^{2}+45 t+14\right) .
$$

Obviously, $J_{4}(t)>0$ for $0<t<1$. This proves the claim.

Hence, $J_{2}^{\prime}(x)>0$ for $0<x<1$. This implies that $J_{2}(x)>J_{2}(0)=0$ and $J_{1}^{\prime}(x)>0$ for $0<x<1$. Therefore, the function $J_{1}(x)$ is strictly increasing on $(0,1)$. And hence,

$$
\frac{10}{3}=\lim _{x \rightarrow 0} J_{1}(x)<J_{1}(x)=\frac{\frac{7}{3}+\sqrt{1-x^{4}}}{x} \operatorname{arctlh} x<\lim _{x \rightarrow 1} J_{1}(x)=\frac{7}{12} B\left(\frac{1}{4}, \frac{1}{4}\right)
$$

for $0<x<1$. Hence, inequality (2.15) holds with the best possible constants given in equation (2.17).

For $0<x<1$, let

$$
T_{1}(x)=\frac{\frac{2}{3}+\left(1-x^{4}\right)^{1 / 4}}{x} \operatorname{arcth} x
$$

Differentiation yields

$$
T_{1}^{\prime}(x)=\frac{2\left(1-x^{4}\right)^{3 / 4}+3}{3 x^{2}\left(1-x^{4}\right)^{3 / 4}} T_{2}(x),
$$


where

$$
T_{2}(x)=-\operatorname{arctlh} x+\frac{x\left(2+3\left(1-x^{4}\right)^{1 / 4}\right)}{2\left(1-x^{4}\right)^{3 / 4}+3} .
$$

Elementary calculation shows that

$$
T_{2}^{\prime}(x)=-\frac{6 x^{4}\left(3-2 \sqrt{1-x^{4}}-\left(1-x^{4}\right)^{3 / 4}\right)}{\left(1-x^{4}\right)^{3 / 4}\left(2\left(1-x^{4}\right)^{3 / 4}+3\right)^{2}}<0, \quad 0<x<1,
$$

which implies that $T_{2}(x)<T_{2}(0)=0$ and $T_{1}^{\prime}(x)<0$ for $0<x<1$. Therefore, the function $T_{1}(x)$ is strictly decreasing on $(0,1)$. And hence,

$$
\frac{1}{6} B\left(\frac{1}{4}, \frac{1}{4}\right)=\lim _{x \rightarrow 1} T_{1}(x)<T_{1}(x)=\frac{\frac{2}{3}+\left(1-x^{4}\right)^{1 / 4}}{x} \operatorname{arctlh} x<\lim _{x \rightarrow 0} T_{1}(x)=\frac{5}{3}
$$

for $0<x<1$. Hence, inequality (2.16) holds with the best possible constants given in equation $(2.18)$

Remark 2.3 There is no strict comparison between the two lower bounds in equations (2.15) and (2.16). Also, there is no strict comparison between the two upper bounds in equations (2.15) and (2.16).

\section{Competing interests}

The authors declare that they have no competing interests.

\section{Authors' contributions}

All authors read and approved the final manuscript.

\section{Acknowledgements}

The authors would like to thank the referees for their careful reading of the manuscript and insightful comments.

Received: 7 July 2013 Accepted: 19 December 2013 Published: 24 Jan 2014

\section{References}

1. Borwein, JM, Borwein, PB: Pi and the AGM: A Study in the Analytic Number Theory and Computational Complexity. Wiley, New York (1987)

2. Carlson, BC: Algorithms involving arithmetic and geometric means. Am. Math. Mon. 78, 496-505 (1971)

3. Neuman, E: On Gauss lemniscate functions and lemniscatic mean. Math. Pannon. 18, 77-94 (2007)

4. Neuman, E: Two-sided inequalities for the lemniscate functions. J. Inequal. Spec. Funct. 1, 1-7 (2010)

5. Neuman, E: On Gauss lemniscate functions and lemniscatic mean II. Math. Pannon. 23, 65-73 (2012)

6. Neuman, E: Inequalities for Jacobian elliptic functions and Gauss lemniscate functions. Appl. Math. Comput. 218 7774-7782 (2012)

7. Neuman, E: On lemniscate functions. Integral Transforms Spec. Funct. 24, 164-171 (2013)

8. Siegel, CL: Topics in Complex Function Theory, vol. 1. Wiley, New York (1969)

9. Mitrinović, DS, Vasić, PM: Analytic Inequalities. Springer, New York (1970)

10. Fink, AM: Two inequalities. Publ. Elektroteh. Fak. Univ. Beogr., Mat. 6, 48-49 (1995)

11. Malešević, BJ: Application of $\lambda$-method on Shafer-Fink's inequality. Publ. Elektroteh. Fak. Univ. Beogr., Mat. 8, 103-105 (1997)

12. Malešević, BJ: One method for proving inequalities by computer. J. Inequal. Appl. 2007, Article ID 78691 (2007). doi:10.1155/2007/78691

13. Malešević, BJ: An application of $\lambda$-method on inequalities of Shafer-Fink's type. Math. Inequal. Appl. 10, 529-534 (2007)

14. Zhu, L: On Shafer-Fink inequalities. Math. Inequal. Appl. 8, 571-574 (2005)

15. Zhu, L: On Shafer-Fink-type inequality. J. Inequal. Appl. 2007, Article ID 67430 (2007). doi:10.1155/2007/67430

16. Pan, W, Zhu, L: Generalizations of Shafer-Fink-type inequalities for the arc sine function. J. Inequal. Appl. 2009, Article ID 705317 (2009). doi:10.1155/2009/705317

17. Zhu, L: A solution of a problem of Oppenheim. Math. Inequal. Appl. 10, 57-61 (2007)

18. Oppenheim, A: E1277. Am. Math. Mon. 64, 504 (1957) 
19. Chen, CP, Cheung, WS: Wilker- and Huygens-type inequalities and solution to Oppenheim's problem. Integral Transforms Spec. Funct. 23, 325-336 (2012)

20. Qi, F, Guo, BN: Sharpening and generalizations of Shafer's inequality for the arc sine function. Integral Transforms Spec. Funct. 23, 129-134 (2012)

21. Qi, F, Guo, BN: Sharpening and generalizations of Shafer-Fink's double inequality for the arc sine function. Filomat 27, 261-265 (2013)

22. Shafer, RE: Problem E1867. Am. Math. Mon. 73, 309 (1966)

23. Shafer, RE: Problems E1867 (solution). Am. Math. Mon. 74, 726-727 (1967)

24. Kuang, JC: Applied Inequalities, 3rd edn. Shangdong Science and Technology Press, Jinan (2004)

25. Qi, F, Zhang, SQ, Guo, BN: Sharpening and generalizations of Shafer's inequality for the arc tangent function. J. Inequal. Appl. 2009, Article ID 930294 (2009). doi:10.1155/2009/930294

26. Chen, CP, Cheung, WS, Wang, W: On Shafer and Carlson inequalities. J. Inequal. Appl. 2011, Article ID 840206 (2011). doi:10.1155/2011/840206

27. Zhu, L: New inequalities of Shafer-Fink type for arc hyperbolic sine. J. Inequal. Appl. 2008, Article ID 368275 (2008). doi:10.1155/2008/368275

28. Chen, CP: Wilker and Huygens type inequalities for the lemniscate functions. J. Math. Inequal. 6, 673-684 (2012)

29. Chen, CP: Wilker and Huygens type inequalities for the lemniscate functions, II. Math. Inequal. Appl. 16, 577-586 (2013)

30. Anderson, GD, Qiu, SL, Vamanamurthy, MK, Vuorinen, M: Generalized elliptic integral and modular equations. Pac. J. Math. 192, 1-37 (2000)

31. Anderson, GD, Vamanamurthy, MK, Vuorinen, M: Conformal Invariants, Inequalities, and Quasiconformal Maps. Wiley, New York (1997)

32. Anderson, GD, Vamanamurthy, MK, Vuorinen, M: Monotonicity rules in calculus. Am. Math. Mon. 113, 805-816 (2006)

33. Lindqvist, P: Some remarkable sine and cosine functions. Ric. Mat. 44, 269-290 (1995)

34. Biezuner, RJ, Ercole, G, Martins, EM: Computing the first eigenvalue of the $p$-Laplacian via the inverse power method. J. Funct. Anal. 257, 243-270 (2009)

35. Bushell, PJ, Edmunds, DE: Remarks on generalised trigonometric functions. Rocky Mt. J. Math. 42, 25-57 (2012)

36. Drábek, P, Manásevich, R: On the closed solution to some $p$-Laplacian nonhomogeneous eigenvalue problems. Differ. Integral Equ. 12,773-788 (1999)

37. Lang, J, Edmunds, DE: Eigenvalues, Embeddings and Generalised Trigonometric Functions. Lecture Notes in Mathematics, vol. 2016. Springer, Berlin (2011)

38. Takeuchi, S: Generalized Jacobian elliptic functions and their application to bifurcation problems associated with p-Laplacian. J. Math. Anal. Appl. 385, 24-35 (2012)

39. Edmunds, DE, Gurka, P, Lang, J: Properties of generalized trigonometric functions. J. Approx. Theory 164, 47-56 (2012)

40. Baricz, A, Bhayo, BA, Klén, R: Convexity properties of generalized trigonometric and hyperbolic functions. Aequ. Math. (2013). doi:10.1007/s00010-013-0222-x

41. Bhayo, BA, Vuorinen, M: On generalized trigonometric functions with two parameters. J. Approx. Theory 164 1415-1426 (2012)

42. Klén, R, Vuorinen, M, Zhang, X: Inequalities for the generalized trigonometric and hyperbolic functions. J. Math. Anal. Appl. 409, 521-529 (2014)

10.1186/1029-242X-2014-35

Cite this article as: Deng and Chen: Sharp Shafer-Fink type inequalities for Gauss lemniscate functions. Journal of Inequalities and Applications 2014, 2014:35

\section{Submit your manuscript to a SpringerOpen ${ }^{\circ}$ journal and benefit from:}

- Convenient online submission

Rigorous peer review

- Immediate publication on acceptance

- Open access: articles freely available online

- High visibility within the field

- Retaining the copyright to your article

Submit your next manuscript at $>$ springeropen.com 